\title{
Modified Fast Bandwidth-Constrained Shortest Path Algorithm for MACO
}

\author{
Amol Parikh \\ Vellore Institute of Technology \\ Vellore, Tamilnadu-632014
}

\author{
Nidhi Sharma \\ Vellore Institute of Technology \\ Vellore, Tamilnadu-632014
}

\author{
J Jeris Alan \\ Vellore Institute of Technology \\ Vellore, Tamilnadu-632014
}

\begin{abstract}
Ant Colony Optimization is a probabilistic technique for finding the optimal path for reaching a destination using graphs. This algorithm was further developed to introduce the concept of Multiple Ant Colony Optimization technique in which ants were classified into various families that worked to provide optimal path solutions to the destination for their own family. This paper aims at proposing an algorithm for reducing the congestion in a network using a modified Fast Bandwidth-Constrained shortest path routing algorithm.
\end{abstract}

\section{Keywords}

MACO - Multiple Ant Colony Optimization

ACO - Ant Colony Optimization

OSPF - Open Shortest Path First

RIP -Routing Information Protocol

\section{INTRODUCTION}

The classic routing algorithms like OSPF, RIP depends upon the information gathered by the routers themselves. With the advancement of networking more complex networks are being developed, newer ways have been found to help construct the routing tables. For example agent-based algorithms are used for solving congestion problems in complex networks.[1][4]One of these agent-basedalgorithms is the Ant Colony Optimization. This algorithm was inspired by the study of biological ants. They wander randomly in search for food but when an ant finds the destination (i.e. the food source) it lays pheromones, a chemical substance that introduces changes to the environment and can be detected by other ants, to the source. This results in other ants following the pheromone trail to the food source. This is a form of indirect communication called Stigmergy [1]. To model the problem solving paradigm of a colony of biological ants, a society of artificial ants(ANTs) are modeled as a society of mobile agents capable of solving various routing and congestion problems. ACO models these ants using mathematical structures and uses them for routing.ACO has been compared with the traditional routing algorithms and has proved better in adapting to changes in the topology, and also has lower routing overhead. The convergence of a path is also found earlier in ACO than in other routing algorithms. [1]Though ACO has several benefits, it has a few disadvantages like congestion and totally abandoning of other paths that have slightly higher cost but could be used when congestion occurs. To solve this problem Multiple Ant Colony Optimization (MACO) was developed. In MACO, the ants were classified into families. Ants of different families have different pheromones that can be detected by ants of the same family only. Hence, all families would try to find different paths and all ants belonging to the same family would follow same path, but ants belonging to different families followed different paths. This somewhat increases adaptiveness and reduces the congestion in the network. However, if the number of ants in a family is very large then stagnation could still occur in the network.

\section{MODIFIED FAST BANDWIDTH- CONSTRAINED SHORTEST PATH ALGORITHM FOR MACO}

In this paper we propose a modification in MACO algorithm. Here, each family of ANTs being considered find their shortest path to the destination using the original Fast Bandwidth- Constrained shortest path algorithm [3]. Once the path has been identified, the ANTs start following the pheromone trail on the path. If the number of ANTs in the family is very large, they might suffer from stagnation at intermediate nodes of the graph. So to eliminate this problem we propose that the header of the ants be modified in such a way that after waiting for a timeout period, the ANTs will be able to detect pheromones of other families as well. The ANTs would then backtrack to the source node and compute the next shortest path amongst the shortest paths of other families.

\subsection{Fast Bandwidth- Constrained Shortest Path Algorithm}

We consider our network to be a graph $\mathrm{G}(\mathrm{V}, \mathrm{E})$ with vertices $\mathrm{V}(\mathrm{G})$ that are the nodes of the network and edges $\mathrm{E}(\mathrm{G})$ which represent the path between these nodes. The Fast BandwidthConstrained shortest path algorithm [3] uses backward routing to start searching for path from the destination node until a shortest path from the source node is found. Here let $\mathrm{s}$ be the source node, and $t$ be the destination node. Also we consider $\mathrm{Q}$ to be the set of nodes yet to be computed and $\mathrm{S}$ be the set of nodes already computed and component of the shortest path. Let $x \quad V(G)$ be the set of nodes in the graph and $\operatorname{Adj}(x)$ be the nodes directly connected to $x$ i.e. $(v, u) \quad E(G)$. According to the Fast Bandwidth-Constrained shortest path algorithm, the distance of all nodes from the destination is set to infinity, and the next of each node is set to nil. After these initializations, all nodes of $\mathrm{Q}$ are considered one after the other. The node being considered is added to the list of computed nodes.[3]The adjacent nodes of the current node that have not been computed yet are considered next. If for the adjacent node y the distance from the destination i.e. $d[y]$ is greater than sum of the weight between itself and the current node $\mathrm{w}(\mathrm{u}, \mathrm{y})$ and distance of $\mathrm{u}$ from the destination node i.e. $\mathrm{d}(\mathrm{u})$ and if the distance of $\mathrm{s}$ is also greater than the sum, then the distance of $y$ from the destination is recomputed as the sum of the weight $\mathrm{w}(\mathrm{u}, \mathrm{y})$ and the distance $\mathrm{d}[\mathrm{u}]$. Also the next node for $y$ is made $u$. [3] 


\subsection{Modified Algorithm}

To this algorithm two functions are added.

The first, in case of congestion, backtracking of the ANT to the source node is required.

Next, the ANT then needs to compute the shortest path among the paths of other families.

for node u $\quad \mathrm{V}(\mathrm{G})$ where ANT needs to backtrack \{

while $(S !=N U L L)$

\{

$d \neg$ extract last element of $\mathrm{S}$

go to $\mathrm{u}$ from $\mathrm{d}$

$\mathrm{u} \neg \mathrm{d}$

\}

\}

Whenever an ANT from a family encounters a congested path on its way to the destination, after a certain time out period, it backtracks to the source. It does so by extracting each element from $\mathrm{S}$, which is the list of already computed nodes until the array is empty. The last extracted node form $\mathrm{S}$ would be the source node.

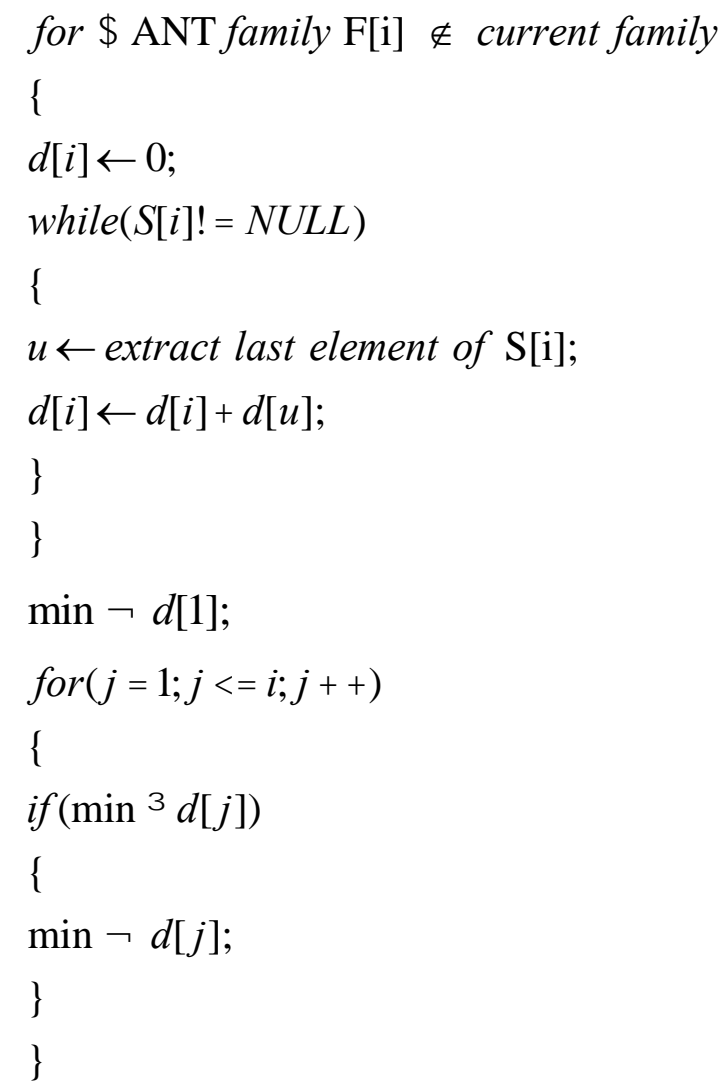
After backtracking back to the source node the ANT now
chooses the shortest path to the destination using the paths of other families. It initializes the distance to zero. Then for each family $\mathrm{i}$ which is not the current family, it calculates the distance to the destination and then chooses a path with minimum distance. The ANT recognizes the pheromone of other families and follows the shortest path of the particular family to its destination.

\subsection{Step - wise Explanation}

Suppose there are three families of ants blue, red and green. Ant of each family is denoted by $\mathrm{A}(\mathrm{b}), \mathrm{A}(\mathrm{r})$ and $\mathrm{A}(\mathrm{g})$ respectively.

Initially all of them are at the starting of the path from the nest to the food source.

Step 1: The ants of different families randomly select the path to reach the food source. $A(b)$ chooses $R 1$ and $R 2$. A(r) chooses R2 and R3 and $\mathrm{A}(\mathrm{g})$ chooses are R4 and R5

Step 2: Since R1 > R2 looking at the pheromone table the ant A(b) will gradually start using only path R2. Similarly the pheromone table for $\mathrm{R} 3$ will be more than $\mathrm{R} 2$ and so $\mathrm{A}(\mathrm{r})$ will using only path $\mathrm{R} 3$ and $\mathrm{A}(\mathrm{g})$ will use only path $\mathrm{R} 4$

Step 3: The ants of each of these three families will now move along these shortest paths only. If the number of ants of any families is higher than the bandwidth of the path, congestion may occur and the ant may not be able to reach the destination(food source). Here an ANT A(b) encounters a congestion on path $\mathrm{R} 2$.

Step 4: After a certain time out period the ant starts backtracking to the source again.

Step 5: After reaching the source the ANT A(b) then chooses the shortest path from the path computed by the ants of the red and green family while ignoring the path computed by the ANTs of its own family i.e blue. Here it finds that the path computed by the red family i.e. R3 is the shortest path to the destination.

Step 6: The ANT A(b) now moves along R3( the shortest path computed by the ants of the red family) thus avoiding the network congestion occurring at R2

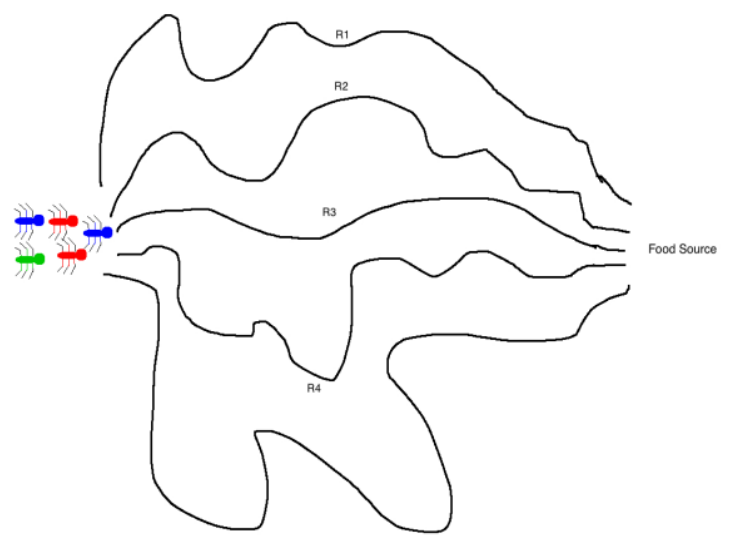

Fig 1 


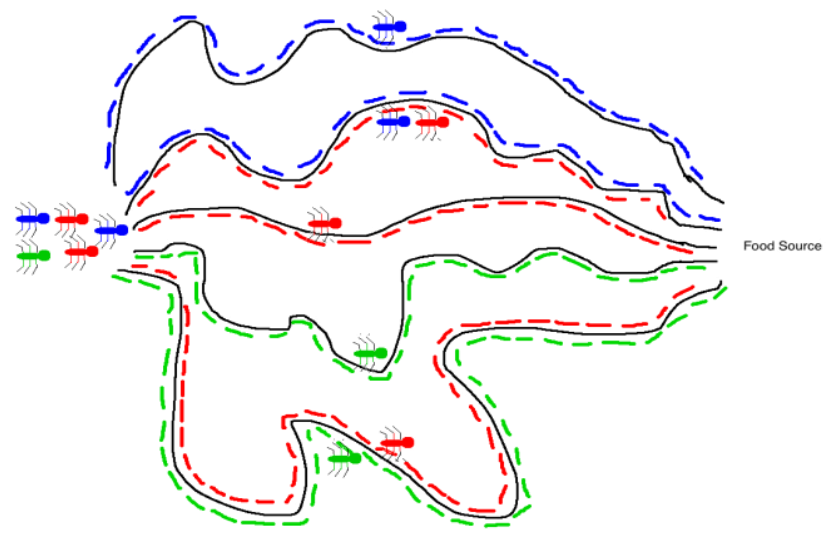

Fig 2

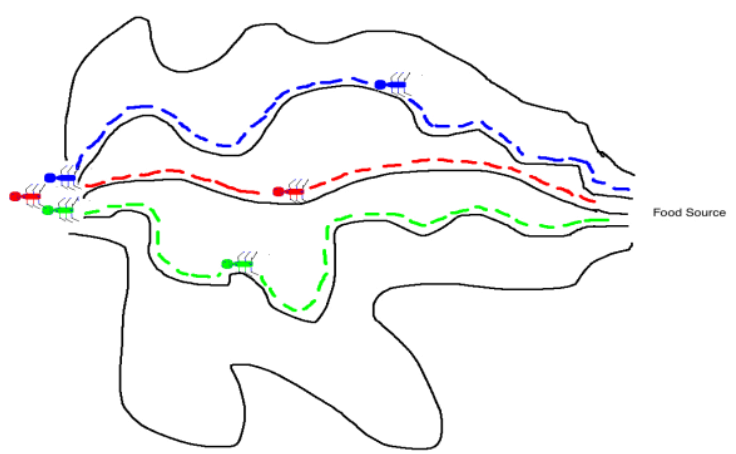

Fig 3

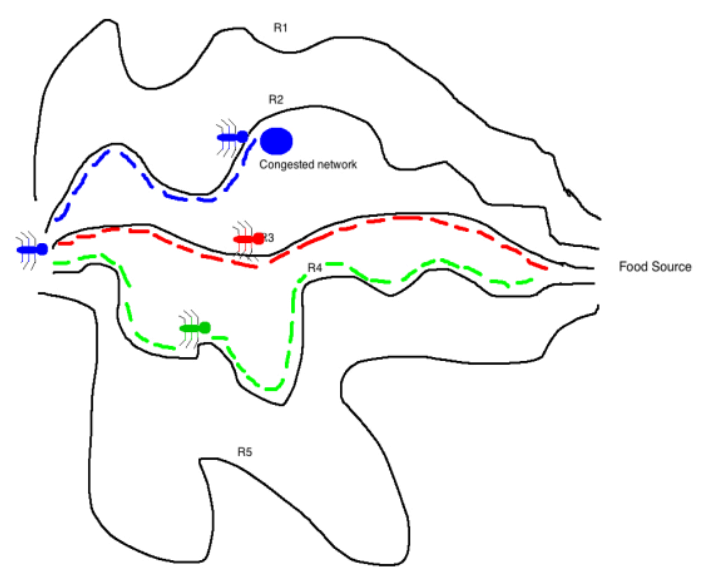

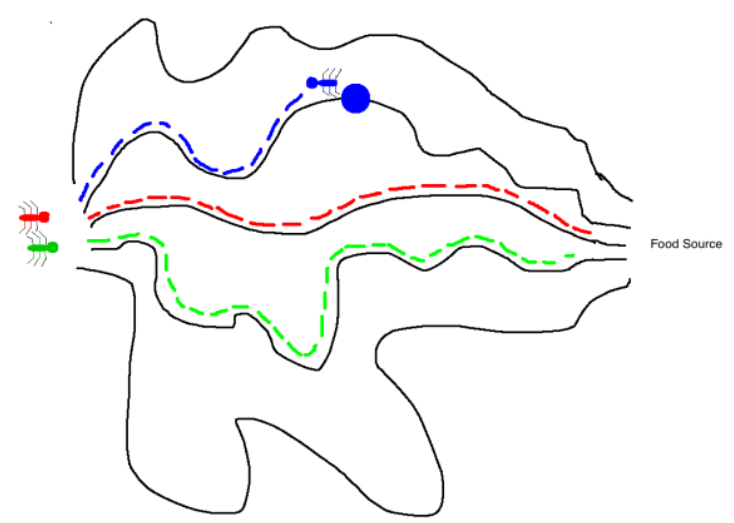

Fig 5

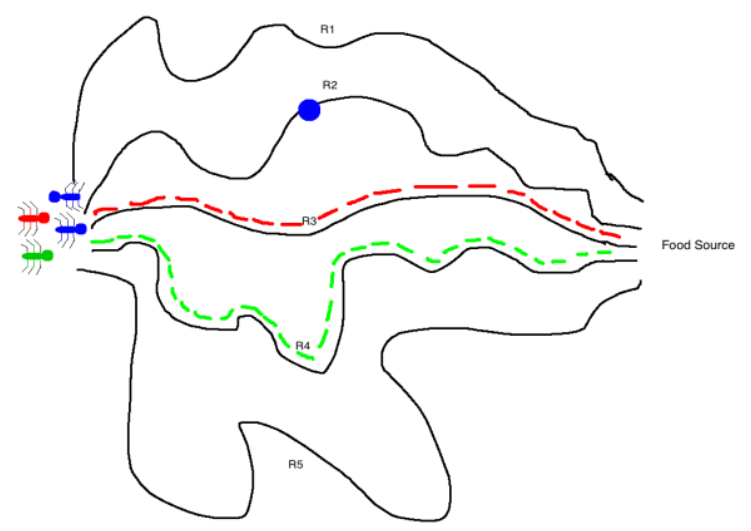

Fig 6

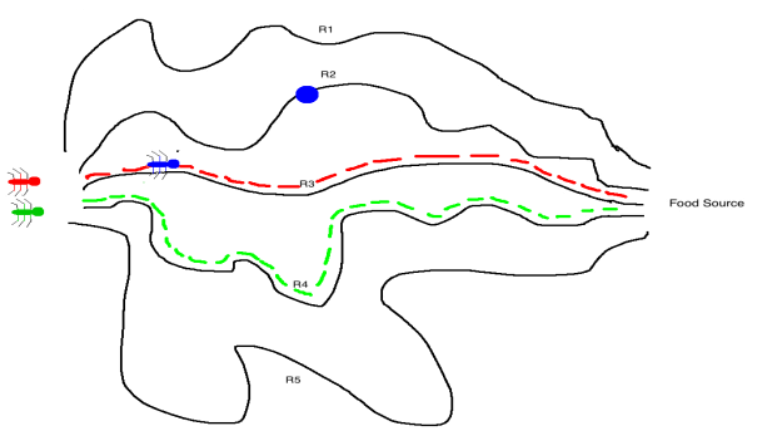

Fig 4

Fig 7 


\section{CONCLUSION}

The Multiple Ant Colony Optimization is used to overcome the shortcomings of Ant Colony Optimization. However, gradually as the number ants of a family that traverse through the network from the source to their destination and back increase, they prefer a single shortest path between the source and destination. As the network traffic increases, the bandwidth utilization increases and the problem of network congestion may arise. If a particular family of ants has a large number of ants traversing through the network, network congestion is bound to occur. The algorithm proposed in this paper is to make the multiple ant colony optimization technique, adapt to such congestion problems. In a dynamic network where the state of a link may change, this algorithm provides a method to adapt to these changes.[1]However in such scenarios the ANT has to backtrack to the source and then choose an alternative path. This eventually reduces the load on a particular path of a graph and eliminates network congestion. However this backtracking results in high network delay and which in turn reduces the throughput of the network. Hence this proposed algorithm eradicates network stagnation and eliminates congestion to a high extent but its implications are high network delay and possibly low guarantee of delivery.

\section{ACKNOWLEDGEMENTS}

We would like to thank Prof. Selvakumar R for his expert advice in the area of Graph theory and its applications in the field of networking.

\section{REFERENCES}

[1] Kwang Mong Sim, Weng Hong Sun, Multiple Ant-Colony Optimization for Network Routing, Proceedings of the First International Symposium on Cyber Worlds (CWí02), IEEE CSE 2002

[2] B. Zhang, Z. Zhao ,H.T. Mouftah, Fast bandwidthconstrained quality of service routing via bidirectional search, The Institution of Engineering and Technology 2007

[3] B. Zhang, H.T. Mouftah , Fast bandwidth-constrained shortest path routing algorithm, The Institution of Engineering and Technology 2006

[4] Kwang Mong Sim and Weng Hong Sun, Ant Colony Optimization for Routing and Load-Balancing: Survey and New Directions, 2003 IEEE

[5] E. Bonabeau, M. Dorigo, and G. Theraulaz, "Inspiration for optimization from social insect behavior," Nature, vol. 406, pp. 39-42, July 2000.

[6] Dorigo, M.; Birattari, M.; Stutzle, T., "Ant colony optimization," Computational Intelligence Magazine, IEEE , vol.1, no.4, pp.28,39, Nov. 2006

[7] Marco Dorigo, Christian Blum., "Ant colony optimization theory : A survey ", Theoratical Computer Science, 30 May 2005

[8] Saad Ghaleb Yaseen, Nada M. A.AL-Slamy, "Ant colony optimization", International Journal of Computer Science and Network Security, VOL. 8No 6. June 2008 